\section{Theoretical study on the need for laser iridotomy in an implantable collamer lens with a hole using computational fluid dynamics}

T Kawamorita ${ }^{1}$, K Shimizu ${ }^{2}$ and N Shoji ${ }^{3}$

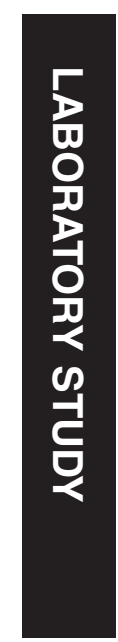
Abstract
Purpose Although one of the advantages of
the Hole-ICL implantation is that laser iridotomy (LI) is unnecessary, the evidence have not been reported from the viewpoint of aqueous humor circulation. We investigated the effect of laser iridotomy (LI) on the fluid dynamics of aqueous humor in an implantable collamer lens (ICL) with a central hole, that is, a Hole-ICL using computational fluid dynamics. Methods A fluid dynamics simulation was performed using the thermal-hydraulic analysis software FloEFD (Mentor Graphics Corp.). For the simulation, three-dimensional eye models with a conventional ICL (Model ICM, STAAR SURGICAL) and a Hole-ICL were used. The LI diameters were 250 and $500 \mu \mathrm{m}$. The flow distribution between the anterior surface of the crystalline lens and the posterior surface of the ICL was also calculated.
Results The flow velocity $0.25 \mathrm{~mm}$ in front of the center of the crystalline lens in the Hole-ICL without LI, with LI of $250 \mu \mathrm{m}$, and with $\mathrm{LI}$ of $500 \mu \mathrm{m}$ was $1.48 \times 10^{-1}, 1.20 \times 10^{-1}$, and $4.52 \times 10^{-2} \mathrm{~mm} / \mathrm{s}$, respectively. The flow velocity in the conventional ICL without $L I$, with LI of $250 \mu \mathrm{m}$, and with LI of $500 \mu \mathrm{m}$ was $1.21 \times 10^{-5}, 3.60 \times 10^{-4}$, and $6.33 \times 10^{-4} \mathrm{~mm} / \mathrm{s}$, respectively.
Conclusions These results suggest that there is less need for LI in a posterior chamber phakic intraocular lens with a central hole from the viewpoint of aqueous humor circulation, although the results can be considered only in an ideal condition and further studies are needed to clarify the effect of LI in clinical practice.
Eye (2017) 31, 795-801; doi:10.1038/eye.2016.279; published online 20 January 2017

\section{Introduction}
Visian Implantable Collamer posterior chamber (PC) phakic intraocular lenses (ICLs, Staar Surgical Co.) have been reported to have many advantages for the treatment of refractive error, especially in cases of high and moderate ametropia. ${ }^{1,2}$ Recently, the toric ICL has been demonstrated to perform well for the correction of moderate or high astigmatism and to provide excellent visual performance. ${ }^{3-5}$ However, one of the major potential complications after ICL implantation is cataract development. ${ }^{6}$ A cause of secondary cataracts is the reduction in the circulation of aqueous humor to the anterior surface of the crystalline lens. Therefore, in 2006, Shimizu created a centrally perforated ICL (ie, the Hole-ICL) to improve aqueous humor circulation (Shimizu K. Implantable Contact Lens. The 31st Annual Meeting of the Japanese Society of Ophthalmic Surgeons, Yokohama, Japan, 2008). Hole-ICL implantation provides good visual performance without loss of optical quality. ${ }^{7-11}$ In addition, one of the advantages of the Hole-ICL implantation is that laser iridotomy (LI) is unnecessary. LI increases the risk of the complications such as iritis, intraocular hemorrhage, among others. ${ }^{12-14}$ In our previous computational fluid dynamics study, ${ }^{15}$ we reported that Hole-ICLs improve the circulation of aqueous humor to the anterior surface of the crystalline lens. However, we did not consider the effect of LI. Therefore, the aim of this study was to investigate the need for LI in a PC phakic intraocular lens with a central hole using computational fluid dynamics. and Visual Science, Kitasato University School of Allied Health Sciences, Kanagawa, Japan

${ }^{2}$ Sanno Hospital Ophthalmology, Tokyo, Japan

${ }^{3}$ Department of Ophthalmology, Kitasato University School of Medicine, Kanagawa, Japan

Correspondence: T Kawamorita, Department of Orthoptics and Visual Science, Kitasato University School of Allied Health Sciences, 1-15-1 Kitasato, Minami-ku, Sagamihara City, Kanagawa, 252-0373, Japan

Tel: +81 42778 9669; Fax: +81427789669. E-mail:kawa2008@ kitasato-u.ac.jp

Received: 13 November 2015 Accepted in revised form: 29 September 2016; Published online: 20 January 2017

Presentation at a conference: An earlier version of this paper was presented at the 116th Annual Meeting of the Japanese Ophthalmological Society, Kyoto, April 6, 2012.
${ }^{1}$ Department of Orthoptics 


\section{Materials and methods}

A computational fluid dynamics simulation of ICL use was performed using the thermal-hydraulic analysis software program FloEFD FE 12.2.0. (Mentor Graphics Corp., Wilsonville, OR, USA), a computational fluid dynamics analysis tool that is commonly embedded in mechanical computer-aided design systems.

For the simulation, three-dimensional eye models with conventional ICLs (Model ICM, STAAR SURGICAL) and Hole-ICLs with and without LI were created (Figures 1 and 2). The eye model in particular has been reported

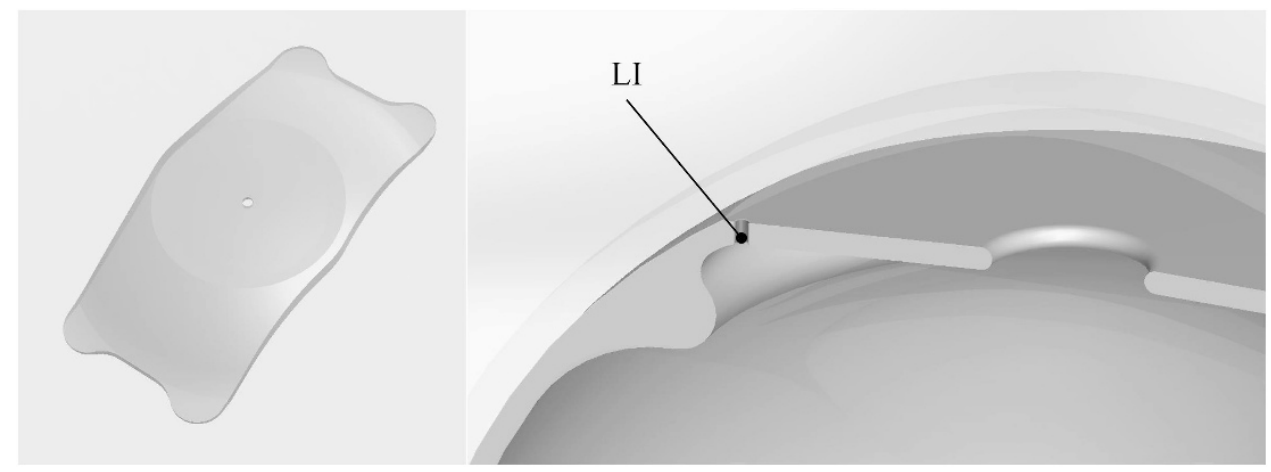

Figure 1 Three-dimensional models of eyes with the Hole-ICL created with FloEFD software. The anterior ocular segment (left) and the location of laser iridotomy (right).

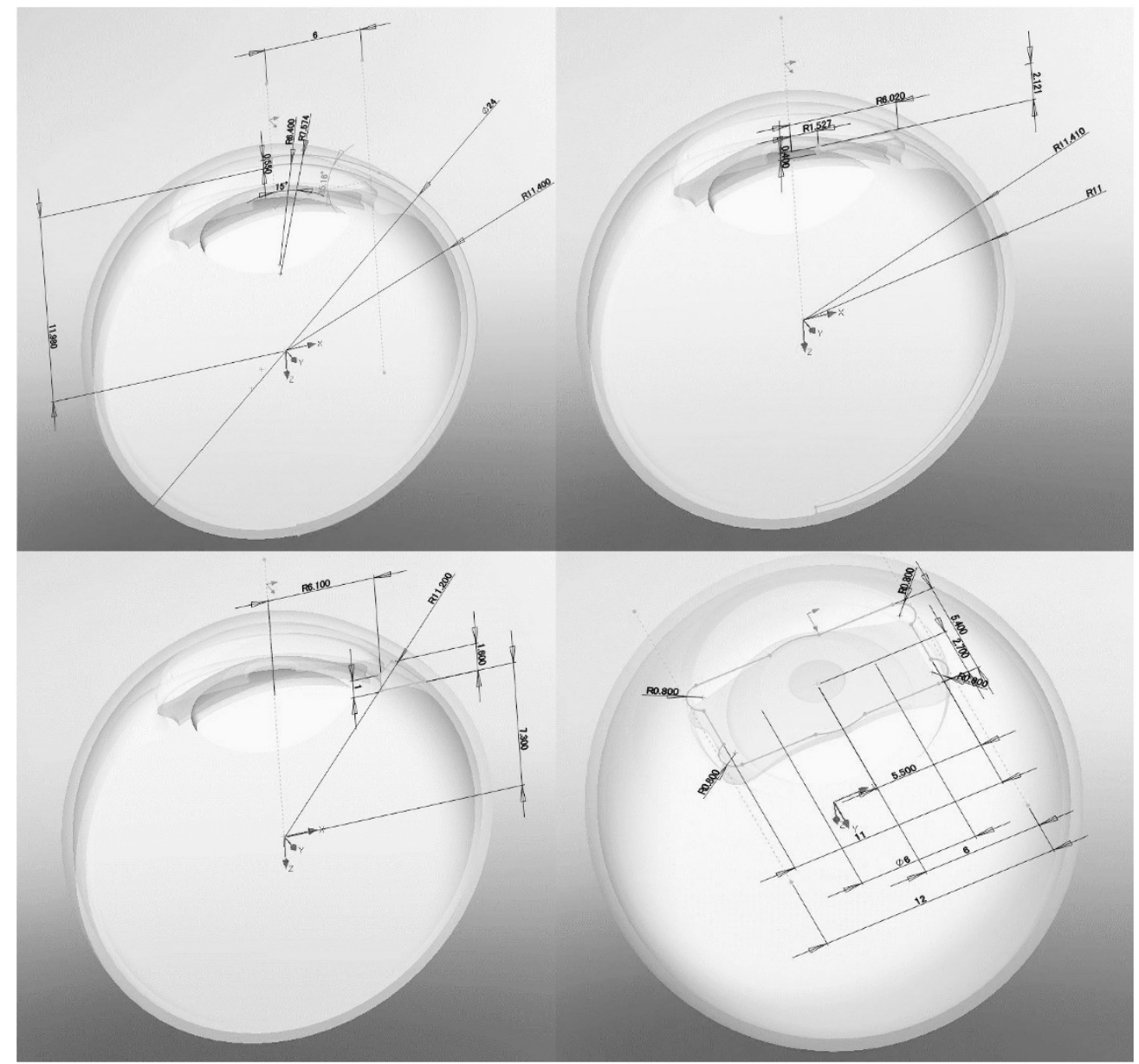

Figure 2 Three-dimensional model setting. 
previously. ${ }^{15}$ Both ICLs were -9.0 diopters (D) and $12.0 \mathrm{~mm}$ in length, with an optic of $5.5 \mathrm{~mm}$ (Figures 1 and 2). The center hole diameter in the Hole-ICL was $0.36 \mathrm{~mm}$. The vaulting was $0.50 \mathrm{~mm}$, the pore space between the posterior iris and the ICL was $0.05 \mathrm{~mm}$, and the angulus iridocornealis was 33 degrees. The quantity of aqueous humor produced by the ciliary body was set at $2.80 \mu \mathrm{l} /$ min (Supplementary Figure 1). ${ }^{16}$ Outflow locations for aqueous humor were set to involve $10 \%$ uveoscleral outflow and $90 \%$ trabecular outflow. The solid-state properties of aqueous humor were set to be similar to those of water; the degree of viscosity of aqueous humor was $7.1917 \times 10^{-4} \mathrm{~Pa} \cdot \mathrm{s}$ at a temperature of 95 degrees Fahrenheit. Also, the details on the meshing has shown in Supplementary Figure. The total number of meshing was 808456 (fluidic cell 179 042; solid cell 345 968; interface cell 283 446; Supplementary Figure 2). The initial pressure was set at 1 atmosphere. Pressure value were calculated at each section. Absolute value of the solution were determined final boundary conditions. The calculation process is standard technique for the software. Also, the diffusion coefficient was added to the momentum equation as follow:

$D=a=\lambda /(\operatorname{Ro} * \mathrm{Cp})$

Where

$D$ : physical diffusion coefficient $\left(\mathrm{m}^{2} / \mathrm{s}\right)$

$a$ : coefficient of thermal diffusivity $\left(\mathrm{m}^{2} / \mathrm{s}\right)$

$\lambda$ : coefficient of thermal conductivity $(\mathrm{W} / \mathrm{m} * \mathrm{~K})$

Ro: dencity $\left(\mathrm{kg} / \mathrm{m}^{3}\right)$

Cp: specific heat $\left(\mathrm{J} / \mathrm{kg}^{*} \mathrm{~K}\right)$

Flow distribution between the anterior surface of the crystalline lens and the posterior surface of the ICL, $0.25 \mathrm{~mm}$ from the center of the crystalline lens (Supplementary Figure 3), was also calculated based on algorithm of the fluid equation and discretization in the software. ${ }^{17}$ In addition, a trajectory analysis was performed for both ICLs.

The LI diameters were 250 and $500 \mu \mathrm{m}$. The number of LIs used was one, and the location was towards ten o'clock (Figure 1). The analyzed cross-section was towards 10 o'clock and contained the center of the LI field.

\section{Results}

The flow distribution between the anterior surface of the crystalline lens and the posterior surface of the Hole-ICL was higher than that between the crystalline lens and the conventional ICL regardless of the presence or absence of LI (Figure 3). The difference was particularly striking in the center. The flow velocity $0.25 \mathrm{~mm}$ in front of the center of the crystalline lens in the Hole-ICL without LI, with LI of $250 \mu \mathrm{m}$, and with LI of $500 \mu \mathrm{m}$ was $1.48 \times 10^{-1}$,
$1.20 \times 10^{-1}$, and $4.52 \times 10^{-2} \mathrm{~mm} / \mathrm{s}$, respectively. That in the conventional ICL without LI, with LI of $250 \mu \mathrm{m}$, and with LI of $500 \mu \mathrm{m}$ was $1.21 \times 10^{-5}, 3.60 \times 10^{-4}$, and $6.33 \times 10^{-4} \mathrm{~mm} / \mathrm{s}$, respectively. In the Hole-ICL with LI, the flow velocity decreased compared with that of the Hole-ICL without LI. The larger the hole diameter of LI was, the slower the flow velocity in front of the center of the crystalline lens was (Figure 3).

The outward flow from the hole in the Hole-ICL was confirmed by trajectory analysis. In the Hole-ICL with LI, aqueous humor was confirmed to flow towards the LI directly, not the void between the ICL and crystalline lens (Figure 4). The inflow toward the LI of the Hole-ICL with LI of $500 \mu \mathrm{m}$ was increased compared with that for LI of $250 \mu \mathrm{m}$ (Figure 5).

\section{Discussion}

Previously, our computational fluid dynamics simulation showed that Hole-ICLs improve the circulation of aqueous humor to the anterior surface of the crystalline lens compared to a conventional ICL. ${ }^{15}$ In an animal study, Fujisawa et al ${ }^{18}$ showed that no cataracts were formed when Hole-ICLs were implanted into porcine eyes. That study concluded that the Hole-ICL allowed sufficient flow of aqueous humor, which was distributed over the anterior surface of the crystalline lens, through its central hole. In addition, Shiratani et al ${ }^{19}$ suggested the possibility of preventing secondary cataracts with the Hole-ICL using minipigs. In clinical results, Shimizu et $a l^{20}$ reported the early clinical outcomes of an ICL with a central hole implantation for 6 months after surgery. No significant increase in intraocular pressure (including a pupillary block) or a secondary cataract occurred in any case during the period of observation. In addition, Hiqueras-Esteban et $a l^{21}$ and Alfonso et $a l^{22}$ did not find significant difference in IOP within or between groups

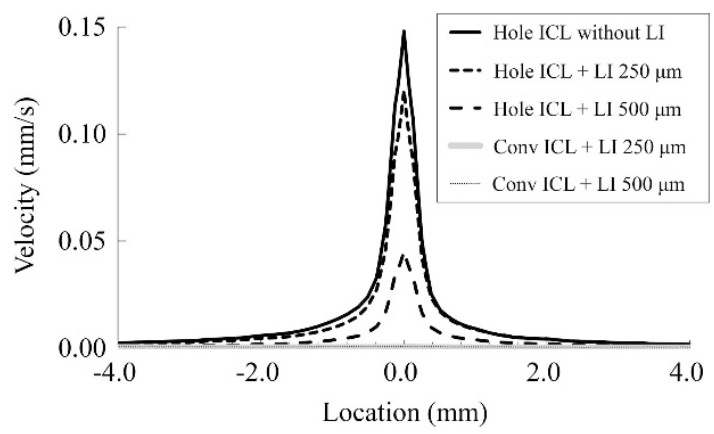

Figure 3 Comparison of flow velocity along the ten o'clock cross-sectional surface of the Hole-ICL without LI and with LI (diameters of 250 and $500 \mu \mathrm{m}$ ) and conventional ICL with LI (diameters of 250 and $500 \mu \mathrm{m}$ ). 
during the follow-up period, although Huseynove et al reported a slightly transient increase (approximately $1 \mathrm{~mm} \mathrm{Hg}$ ) in IOP at 1 week and 1 month postoperatively. Therefore, Hole-ICLs are a beneficial correction method that decreases the risk of secondary cataracts.

In this study, we investigated the effect of LI on the dynamic characteristics of aqueous humor in a Hole-ICL using computational fluid dynamics. The results show that the flow velocity in the conventional ICL was likely not increased because the outflow of the aqueous humor was cut off midstream. A significant quantity of aqueous humor passed through the central hole when the HoleICL was implanted because the distance between the iris and ICL was short. In addition, the increase in the LI diameter was conducive to the decrease in the flow velocity of aqueous humor around the crystalline lens, and the Hole-ICL without LI maintained the good circulation compared with the Hole-ICL with LI because aqueous humor in the Hole-ICL with LI directly flowed out from the ciliary body to the LI location. Many surgeons perform peripheral LI prior to ICL implantation to prevent a pupillary block. LI may increase the risk of complications such as iritis, intraocular hemorrhage, elevation of intraocular pressure, posterior iris synechia, and corneal decompensation, such as bullous keratopathy. ${ }^{12-14}$ Originally, Hole-ICLs have been recommended without LI, and our results suggest that LI is unnecessary from the viewpoint of theoretical aqueous circulation. Although further studies are needed to clarify these effects in vivo, the use of the Hole-ICL may improve aqueous humor circulation, preventing secondary cataracts on a long-term basis and eliminating the need for LI.

Regarding the limitations of the present study, this study was conducted via computer simulation using the standard model eye. The results cannot be generalized, it can be considered only in an ideal condition in terms of natural lens conditions, placement of ICL in terms of vault and sizing and physiological condition of aqueous and its flow. We did not consider the individual differences in anatomical structure, the flow distribution in the posterior eye, ${ }^{23}$ and individual differences that would affect the absolute value of the flow velocity. However, the agreement between theory and the experimental data were high, as shown in the technical references, and based on the validity of the FloEFD software, which utilizes computational fluid dynamics. ${ }^{17}$ As for individual differences, we think that the impact would not change the conclusion in the presence or absence of LI. In

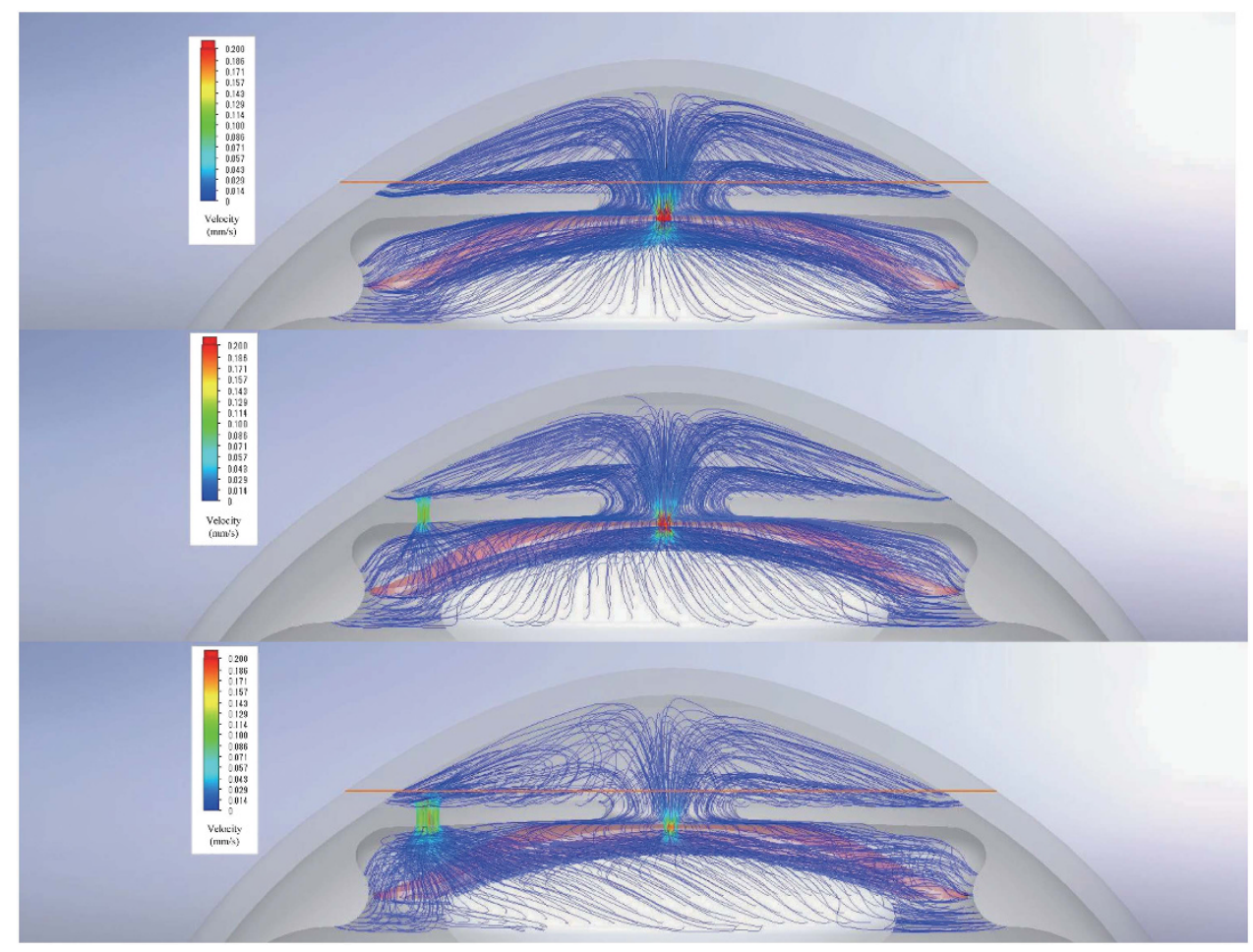

Figure 4 Trajectory analysis in a Hole-ICL without LI (top), with LI of $250 \mu \mathrm{m}$ (middle), and with LI of $500 \mu \mathrm{m}$ (bottom) along the 10 o'clock cross-sectional surface. 


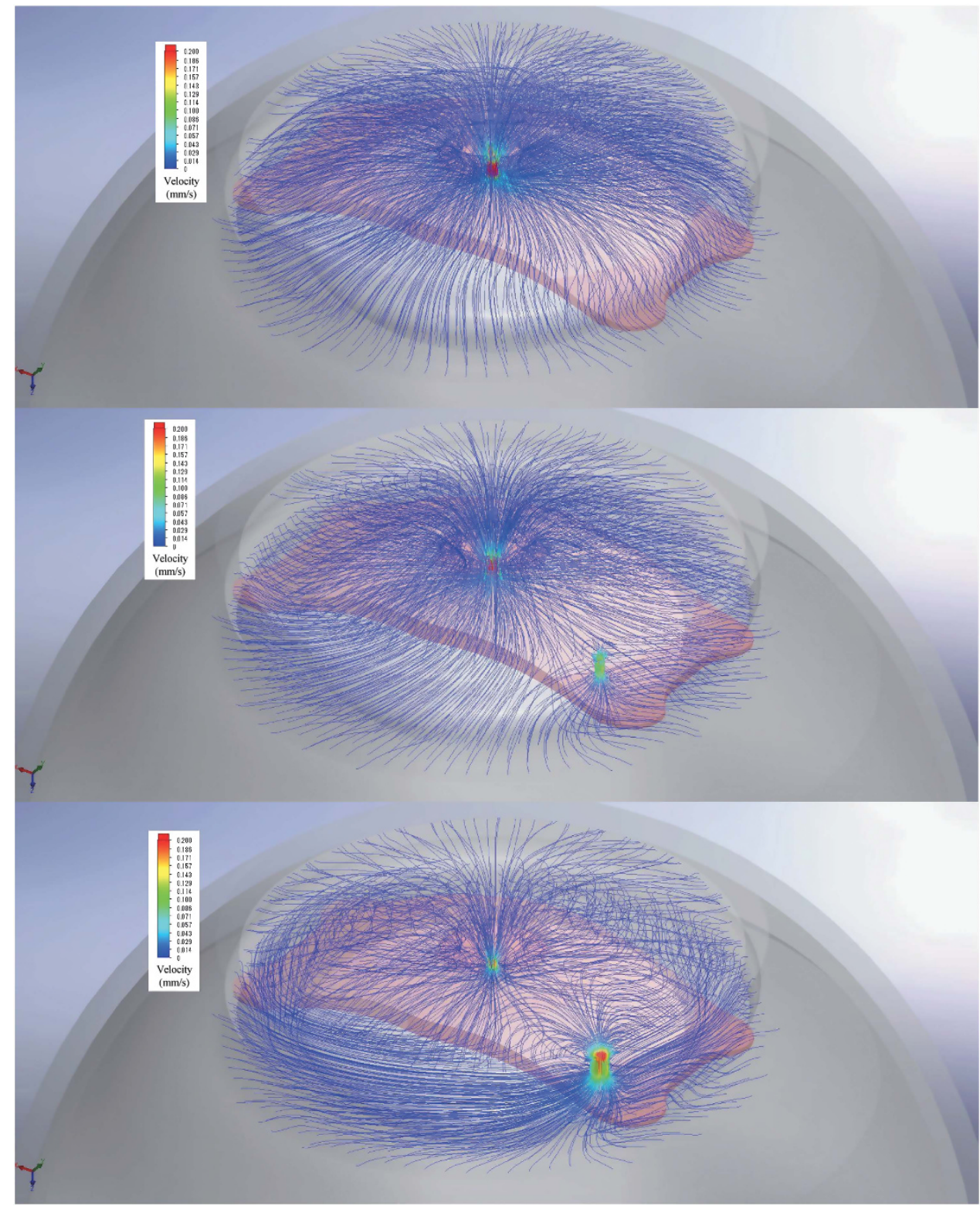

Figure 5 Trajectory analysis in a Hole-ICL without LI (top), with LI of $250 \mu \mathrm{m}$ (middle), and with LI of $500 \mu \mathrm{m}$ (bottom).

addition, although we confirmed the effect of LI in the Hole-ICL, the flow may be increased in association with the pumping action occurring due to physiological changes in the crystalline lens and/or pupil.

Further studies are needed to clarify the effect of LI on corneal endothelium and that in animal model.

In conclusion, these results would make the laser iridotomy less necessary in a PC phakic intraocular lens with a central hole from the viewpoint of aqueous humor circulation, although the results cannot be generalized and can be considered only in an ideal condition.
Summary

What was known before

- In our previous computational fluid dynamics study, we reported that Hole-ICLs improve the circulation of aqueous humor to the anterior surface of the crystalline lens. However, we did not consider the effect of LI.

\section{What this study adds}

- These results suggest that there is less need for LI in a posterior chamber phakic intraocular lens with a central hole from the viewpoint of aqueous humor circulation, although the results can be considered only in an ideal condition and further studies are needed to clarify the effect of LI in clinical practice. 


\section{Conflict of interest}

Dr Shimizu is a paid consultant of STAAR Surgical who has assisted with the development of patented technologies. The remaining authors declare no conflict of interest.

\section{Author contributions}

Involved in conception and design of the study (TK, KS, and NS); Data collection (TK); Analysis and interpretation (TK); Writing the article (TK); Critical revision of the article (TK, KS, and NS); Administrative, technical, and material support (KS); Supervision (KS and NS). The authors had full access to all the data in the study and take responsibility for the integrity of the data and the accuracy of the data analysis.

\section{Acknowledgements}

We thank Dr Hiroshi Uozato for teaching and encouragement, and KOZO KEIKAKU ENGINEERING, Inc., (Mr Osamu Kuwahara and Dr Tsuyoshi Yamada) for technical support and Nature Publishing Group Language Editing for the critical reading of the manuscript. This study was supported by a grant from the Kitasato University School of Allied Health Sciences (Grant-in-Aid for Research Project, No. 2015-1029) (TK), a Kitasato University Research Grant for Young Researchers (2013, 2014, 2015, and 2016) (TK), and a Grant-in-Aid for Young Scientists (B) (TK).

\section{References}

1 Chen LJ, Chang YJ, Kuo JC, Rajagopal R, Azar DT. Metaanalysis of cataract development after phakic intraocular lens surgery. J Cataract Refract Surg 2008; 34: 1181-1200.

2 Lovisolo CF, Reinstein DZ. Phakic intraocular lenses. Surv Ophthalmol 2005; 50: 549-587.

3 Kamiya K, Shimizu K, Kobashi H, Igarashi A, Komatsu M. Three-year follow-up of posterior chamber toric phakic intraocular lens implantation for moderate to high myopic astigmatism. PLoS One 2013; 8: e56453.

4 Kamiya K, Shimizu K, Kobashi H, Igarashi A, Komatsu M, Nakamura A et al. Three-year follow-up of posterior chamber toric phakic intraocular lens implantation for the correction of high myopic astig-

matism in eyes with keratoconus. Br J Ophthalmol 2015; 99: 177-183.

5 Sanders DR, Schneider D, Martin R, Brown D, Dulaney D, Vukich J et al. Toric Implantable Collamer Lens for moderate to high myopic astigmatism. Ophthalmology 2007; 114: 54-61.

6 Fernandes P, Gonzalez-Meijome JM, Madrid-Costa D, Ferrer-Blasco T, Jorge J, Montes-Mico R. Implantable collamer posterior chamber intraocular lenses: a review of potential complications. J Refract Surg 2011; 27: 765-776.
7 Kamiya K, Shimizu K, Saito A, Igarashi A, Kobashi H. Comparison of optical quality and intraocular scattering after posterior chamber phakic intraocular lens with and without a central hole (Hole ICL and Conventional ICL) implantation using the double-pass instrument. PLoS One 2013; 8: e66846.

8 Ohmoto F, Shimizu K, Uozato H, Kawamorita T, Uga S. Optical performances of Implantable Collamer Lenses with and without a central perforation. Kitasato Med J 2010; 40: 150-153.

9 Perez-Vives C, Ferrer-Blasco T, Madrid-Costa D, Garcia-Lazaro S, Montes-Mico R. Optical quality comparison of conventional and hole-visian implantable collamer lens at different degrees of decentering. Am J Ophthalmol 2013; 156: 69-76 e1.

10 Shimizu K, Kamiya K, Igarashi A, Shiratani T. Intraindividual comparison of visual performance after posterior chamber phakic intraocular lens with and without a central hole implantation for moderate to high myopia. Am J Ophthalmol 2012; 154: 486-94 e1.

11 Uozato H, Shimizu K, Kawamorita T, Ohmoto F. Modulation transfer function of intraocular collamer lens with a central artificial hole. Graefes Arch Clin Exp Ophthalmol 2011; 249: 1081-1085.

12 Drake MV. Neodymium:YAG laser iridotomy. Surv Ophthalmol 1987; 32: 171-177.

13 Quigley HA. Long-term follow-up of laser iridotomy. Ophthalmology 1981; 88: 218-224.

14 Siam GA, de Barros DS, Gheith ME, Batiste CG, Tittler EH, Moster MR et al. Post-peripheral iridotomy inflammation in patients with dark pigmentation. Ophthalmic Surg Lasers Imaging 2008; 39: 49-53.

15 Kawamorita T, Uozato H, Shimizu K. Fluid dynamics simulation of aqueous humour in a posterior-chamber phakic intraocular lens with a central perforation. Graefes Arch Clin Exp Ophthalmol 2012; 250: 935-939.

16 Brubaker RF. Flow of aqueous humor in humans [The Friedenwald Lecture]. Invest Ophthalmol Vis Sci 1991; 32: 3145-3166.

17 SolidWorks Flow Simulation 2012 Technical Reference. In: SolidWorks, (ed.). SolidWorks Flow Simulation 2012 Technical Reference, 2012. Available at: https:// d2t1xqejof9utc.cloudfront.net/files/18565/SW_CFD_ technical_reference.pdf?1361897013.

18 Fujisawa K, Shimizu K, Uga S, Suzuki M, Nagano K, Murakami $Y$ et al. Changes in the crystalline lens resulting from insertion of a phakic IOL (ICL) into the porcine eye. Graefes Arch Clin Exp Ophthalmol 2007; 245: $114-122$.

19 Shiratani T, Shimizu K, Fujisawa K, Uga S, Nagano K, Murakami Y. Crystalline lens changes in porcine eyes with implanted phakic IOL (ICL) with a central hole. Graefes Arch Clin Exp Ophthalmol 2008; 246: 719-728.

20 Shimizu K, Kamiya K, Igarashi A, Shiratani T. Early clinical outcomes of implantation of posterior chamber phakic intraocular lens with a central hole (Hole ICL) for moderate to high myopia. Br J Ophthalmol 2012; 96: 409-412.

21 Higueras-Esteban A, Ortiz-Gomariz A, Gutierrez-Ortega R, Villa-Collar C, Abad-Montes JP, Fernandes P et al. Intraocular pressure after implantation of the Visian Implantable Collamer Lens With CentraFLOW without iridotomy. Am J Ophthalmol 2013; 156: 800-805. 
22 Alfonso JF, Lisa C, Fernandez-Vega Cueto L, Belda-Salmeron L, Madrid-Costa D, Montes-Mico R. Clinical outcomes after implantation of a posterior chamber collagen copolymer phakic intraocular lens with a central hole for myopic correction. J Cataract Refract Surg 2013; 39: 915-921.
23 Park J, Bungay PM, Lutz RJ, Augsburger JJ, Millard RW, Sinha Roy A et al. Evaluation of coupled convective-diffusive transport of drugs administered by intravitreal injection and controlled release implant. J Control Release 2005; 105: 279-295.

Supplementary Information accompanies this paper on Eye website (http://www.nature.com/eye) 